KIT FINE

\title{
CRITICAL REVIEW OF PARSONS' NON-EXISTENT \\ OBJECTS *
}

(Received 9 August, 1982)

There has recently been a rebellion within the ranks of analytic philosophy. It has come to be appreciated that, in the debate between Russell and Meinong, Russell was perhaps mistaken in his criticisms and Meinong was perhaps correct in his views. As a consequence, an attempt was made to rehabilitate the Meinongian position, to defend it against the most obvious attacks and to develop it in the most plausible ways. T. Parsons was among the first of the contemporary philosophers to make this attempt, ${ }^{1}$ and so it is especially appropriate that his views should now be set out in a book.

I should say, at the outset, that I thoroughly approve of the Meinongian project. As Parsons makes clear (pp. 32-38), we refer to non-existents in much the same way as we refer to other objects. It is therefore incumbent upon the philosopher to work out the principles by which our discourse concerning such objects is governed. Not that this is necessarily to endorse a realist position towards the objects of the resulting theory. Nominalists and Platonists alike may attempt to set out the principles.that govern arithmetical discourse; and it is in the same spirit that the realist or anti-realist may attempt to set out the principles of our fictional discourse.

Despite my approval of the project, I must admit to some misgivings as to how Parsons has carried it out. These misgivings are of two kinds. There are first some internal criticisms, requiring only change within Parsons' basic approach. There are then some external criticisms, requiring change to the basic approach.

These criticisms, though, should not be thought to detract from the merits of Parsons' book. It is, in many ways, an admirable contribution to the field. It gives weight both to the interest and the legitimacy of the Meinongian enterprise; it pinpoints the difficulties which any satisfactory theory must deal with; and in its solution to those difficulties, it sets up a theory with a degree of rigour and systematicity that should serve as a model for years to 
come. As a well worked-out and accessible contribution to object theory, there is no better book.

\section{BACKGROUND}

Before presenting Parsons' views, it will be worthwhile to place his approach to object theory in perspective. The theory of objects may be seen to have its origin in the following naive principle of abstraction:

For any condition on properties, there is an object that has exactly those properties satisfying the condition.

In the same way, the theory of properties or of sets might be seen to have its origin in the principle:

For any condition on objects, there is a property (set) that has (or contains) just those objects satisfying the condition.

There are those for whom this is an inauspicious start to any theory. What distinguishes properties from objects, they would say, is just that there are complex properties - the complement of red, the disjunction of red or green - but not, in the same way, complex objects - the 'complement' of Socrates, the 'disjunction' of Socrates and Plato. I would not, in the last analysis, wish to endorse anything quite like the naive abstraction principle for objects, but such philosophers would do well to reflect on the grounds for discriminating between objects and properties in this way. There is a complete symmetry in the two forms of abstraction principle. In the one case, the domain of objects is kept fixed and properties are introduced to enter into new configurations of relationship with those objects. In the other case, the domain of properties is kept fixed and objects are introduced to enter into new configurations of relationship with the properties. What grounds are there, then, for preferring the one kind of principle of abstraction or domain extension to the other?

But even if the abstraction principles are allowed to stand, there will be problems with the naive approach. In the first place, both abstraction principles will be internally inconsistent. If the 'objects' are taken to include the properties, then property abstraction will lead to paradoxes of the sort described by Russell; and object abstraction will lead to similar paradoxes should the properties be taken to include the objects. Indeed, in this respect, 
object abstraction has something of an edge over property abstraction; for whereas it is plausible to include the properties among the objects, it is not so plausible to include the objects among the properties.

But even if the paradoxes could somehow be resolved, the two principles would still be inconsistent when taken together. Perhaps the simplest case of inconsistency arises from using property abstraction to obtain a property, such as being self-distinct, not had by any object, and then using object abstraction to obtain an object which has that property.

The domain of objects and properties cannot be extended in the ways jointly prescribed by the naive principles of object and property abstraction. The naive theory must be modified.

There are basically two ways in which this can be done, both suggested by Meinong's pupil, Mally. ${ }^{2}$ The first depends upon introducing two copulas: one is an ordinary copula and may be called 'exemplification'; the other is a special copula, which we may follow Zalta [9] in calling 'encoding'. The principle of property abstraction will then be used to state the conditions under which an object exemplifies a property, the principle of object abstraction to state the conditions under which an object encodes a property. The obvious sources of conflict between the two principles will then be removed. The object that has the (or a) null property, for example, will encode that property but not exemplify it.

The second method depends upon introducing two kinds of property: the ordinary or 'nuclear' properties, and the special or 'extra-nuclear' ones. The properties assumed to exist under property abstraction will be extranuclear, while the properties used to characterize objects under object abstraction will be nuclear. The null property will then be extra-nuclear and so will not be had by any object.

Very roughly, we may say that Castañeda [1], Rappaport [7] and Zalta [9] favour the dual copula approach, whilst Parsons and Routley [8] favour the dual property approach. It is rather hard to classify the theory considered in my [2], since its story-relative copula is not properly a copula at all. But formally, it is closest to the dual copula approach.

As is only natural at this stage of enquiry, advocates of either approach have been concerned to develop their own particular theories. But now that those theories are at hand, what is badly needed is some sort of overview. One is reminded of the situation that prevailed in set theory prior to the advent of proof- and model-theoretic methods of comparison. One then had a plethora 
of set theories, each with its own motivation and notation - type theory, Zermelo-Fraenkel set theory, set/class theory, and so on. We now have a much better understanding of the connections among these different theories; and it is to be hoped that a similar understanding can be gained for the different object theories.

On the face of it, the two approaches to the subject are very different, with the one postulating a fundamental ambiguity in the copula and the other a fundamental division within the category of properties. But there is a way in which the two approaches can be brought closer together. We may treat the encoder's assertion that $x$ exemplifies $\boldsymbol{P}$ as tantamount to the nuclear theorist's assertion that $x$ has $\boldsymbol{P}$; and we may treat the encoder's assertion that $x$ encodes $\boldsymbol{P}$ as tantamount to the nuclear theorist's assertion that $x$ has the nuclear property $\boldsymbol{P}^{N}$ associated with $\boldsymbol{P}$ (of which more will be said later). Under the reasonable assumption that every nuclear relation is a nuclear weakening $\boldsymbol{P}^{N}$ of some extra-nuclear relation $\boldsymbol{P}$, this then leads to a two-way translation between the languages of the encoder and the nuclear-theorist.

The difference between the two might be put in the following way. Each subject-predicate statement is first expressed in neutral fashion as $(P, t, \pi)$, where $\pi$ indicates the status of the predication as 'ordinary' or 'special'. The encoder then thinks of the status-indicator as attaching to the copula in ordinary subject-predicate statements, while the nuclear theorist conceives of it as attaching to the predicate.

Under such a translation, the theories on one approach will be interpretable as theories on the other approach. It is not to be expected that the actual theories developed on either approach will be mutually interpretable; the choice of the axioms is too random for that. But to any reasonable theory of the one sort, there will correspond a reasonable theory of the other sort.

The translation might also be used to set up a correspondence between the problems that arise on either approach and the solutions that may be given to them. It will be recognized that the various problems are not peculiar to either approach, but will have their counterpart for the other.

Of course, whenever one has the mutual interpretability of two theories, the question arises as to which, if either, is more basic. Is talk of encoding merely an indirect way of referring to the nuclear weakening $P^{N}$, or nuclear weakening an indirect way of referring to encoding? Or are both perhaps mere notational variants of some more basic language?

I must admit, given that one is going to talk either language, to having 
some preference for the encoder's way of putting things. Even if one grants that the ordinary copula is unambiguous, it seems almost irresistible to suppose that the way a non-existent has a nuclear property is different from the way an existent has that property or any object has an extra-nuclear property. The very word 'nuclear' suggests as much. It is as if the nuclear property were internal to its non-existent bearer, but external to its existent bearer - somehow constituting the non-existent, but not the existent. But if this is so, it would suggest that the ordinary copula is to be analysed in to two relations of the sort favoured by the encoder, with the one serving to say how the object is defined and the other how it is characterised.

\section{THE THEORY}

At the heart of Parsons' theory is a distinction and two principles. The distinction is between nuclear and extra-nuclear properties. Ordinary properties such as being a detective, being golden, being a mountain - are nuclear; the others are extra-nuclear (pp. 22-23). The distinction may also be understood in terms of its role in the theory. Nuclear properties are used to formulate the principle of object abstraction, extra-nuclear properties the principle of property abstraction. Nuclear properties, we see, serve to define non-existents, extra-nuclear properties to qualify them.

One of the two principles is a restricted version of Abstraction. It states:

For any condition on nuclear properties, there is an object having exactly those nuclear properties satisfying the condition (pp. 19, 73).

Given that being golden and being a mountain are nuclear, it follows from this principle that there is an object whose sole nuclear properties are being golden and being a mountain. It also follows that there is an object, a 'complement' of Socrates, whose nuclear properties are exactly those not possessed by Socrates.

The other principle is for the identity of objects. It states:

Objects with the same nuclear properties are the same (pp. 19, 74).

It follows from this principle that there is at most one object whose nuclear properties are being golden and being a mountain or at most one object which 
is the complement of Socrates. Thus from the two principles together, it follows that there is a unique object whose nuclear properties are being golden and being a mountain ('the golden mountain') and that there is a unique complement to Socrates.

This is the core theory. It gets extended in two main ways - first to nuclear weakenings, then to nuclear relations. For the first extension, Parsons supposes that for any extra-nuclear property or relation $\boldsymbol{R}$, there is a nuclear weakening or watered-down version $R^{N}$ of $R^{3}$ The nuclear weakening will agree with the original relation on existents, i.e.

For any existents $x_{1}, \ldots, x_{n}, R^{N}$ of $x_{1} \ldots x_{n}$ iff $R$ of $x_{1} \ldots x_{n}$ (p. 73).

The advantage of the nuclear weakening over its original is that it may be used to define new objects. But in order that there should be a reasonable stock of extra-nuclear relations and hence of weakenings, Parsons assumes a suitable form of relation abstraction:

For any condition on objects, there is an extra-nuclear relation relating exactly those objects (in the right order) that satisfy the condition (p. 72).

For example, from this principle there is an extra-nuclear property had by exactly those objects that are not round; and so by the weakening principle, there is a nuclear property had by those existents that are not round.

Parsons later assumes that there is a specific extra-nuclear property $\lambda x \varphi(x)$, the property of $x$ 's $\varphi$-ing, that conforms to property abstraction for the condition $\varphi(x)$ and (similarly for relations). So by weakening, there will be a specific nuclear property $(\lambda x \varphi(x))^{N}$ that is had by an existent just in case it $\varphi s$.

Parsons spends a good deal of time on explaining the nuclear/extra-nuclear distinction, but comparatively little on the concept of nuclear weakening. This associated concept is perhaps best understood in terms of two notions of property abstraction. The property $\lambda x \varphi(x)$ of being a $\varphi$-er may be understood to conform to the unrestricted principle of property abstraction, or it may be understood to conform to property abstraction in its application to existents, but to be at the mercy of object abstraction in its application to non-existents. The one property may be denoted by $\lambda x \varphi(x)$ and the other by $\lambda^{N} x \varphi(x)$. The nuclear weakening $(\lambda x \varphi(x))^{N}$ of $\lambda x \varphi(x)$ may then be 
taken to be $\lambda^{N} x \varphi(x){ }^{4}$ This account conforms to what Parsons himself says, since he defines $\lambda^{N} x \varphi(x)$ as $(\lambda x \varphi(x))^{N}$ (p. 104), but not to his order of explanation.

To develop the theory of nuclear relations, Parsons introduces the notion of plugging. He supposes that from any $(n+1)$-place nuclear (extra-nuclear) relation, $n>1$, an $n$-place nuclear (extra-nuclear) relation can be obtained by plugging up one of its argument-places with an object (p. 65). Thus if the given relation is the 2-place nuclear relation $R$, then two nuclear properties may be obtained by plugging, one the property $[R x]$ of bearing $R$ to $x$ and the other the property $[x R]$ of being an object to which $x$ bears the relation $R$. If, for example, $R$ is the relation of kicking and $x$ is Holmes, then $[R x]$ is the property of kicking Holmes, while $[x R]$ is the property of having Holmes kick one.

The assumptions concerning the operation of plugging are for the most part straightforward. However, two are of particular interest. The first states that no existent has the nuclear property $[R x]$ of bearing a nuclear relation $R$ to a non-existent $x$, and similarly for other plug-ups (pp. 75-76). It is impossible by this assumption, for example, that Gladstone should have the property of kicking Holmes, though Watson may have that property. According to the second of the two assumptions (which is put forward as a definition), $x$ bears the nuclear relation $R$ to $y$ if and only if $x$ has the property $[R y]$ and $y$ has the property $[R x]$, i.e. $x R y \equiv x[R y] \wedge[x R] y$ (p. 66). It follows from this, for example, that Watson kicks Holmes iff Watson has the property of kicking Holmes and Holmes has the property that Watson kicks him.

The analysis of relations is one of the most distinctive features of Parsons' theory; though, in some respects, it is reminiscent both of the early attempt to fit relational statements within the framework of Aristotelian logic and the more recent reduction by Schönfinkel of many-place to one-place functions.

As the theory stands, it is unclear how it is to apply to non-existents as they are ordinarily given in stories, beliefs, dreams, and the like. To this end, Parsons introduces what I call the Link Hypothesis. Let us treat of stories as a typical case. Say that an object of a story is native if it is introduced or created in that story, and otherwise immigrant. For example, Rosencrantz is native to Shakespeare's Hamlet but not to the Tom Stoppard play. Then the Link Hypothesis states that: 
An object native to a story has exactly those nuclear properties attributed to it in the story (pp. 54-55).

Thus this hypothesis effects a bridge between the world of the story and the world of reality.

Given the hypothesis, we may apply the theory to those fictional characters that we ordinarily talk about. Rosencrantz, for example, will have various nuclear properties in Hamlet; and so he will be that object whose nuclear properties are those that are attributed to Rosencrantz in the play.

The above theory is intended to solve broadly three main problems. The first is to account, or allow, for the truth of many of our ordinary judgements concerning non-existents - that Holmes is a detective, that Pegasus is a mythical horse, that Holmes is more famous than any real detective (cf. p. 32). A certain sub-class of these judgments is of particular importance. They include 'Holmes is a detective' and 'Hamlet is a prince' and are true, if true at all, because of what is said in the story or context in which the objects appear. Such judgements may be called literalist.

The second problem is to explain what nonexistents there are, to provide a reasonably rich ontology of such objects. The final problem is to explain what those objects are, to provide some method of individuation for them. The last two problems might be called, somewhat grandiosely, the problems of ontology and identity (cf. p. 52).

The first of the problems will only arise for those who accept the truth of the judgements in question. On the other hand, the other two problems are likely to arise for anyone who takes our reference to non-existents seriously.

We can see, in broad terms, how Parson's theory solves these problems. First, the Link Hypothesis will tell us why Hamlet is a prince or Holmes a detective. Second, Object Abstraction will tell us what objects there are, viz one for each class of nuclear properties. Finally, Object Identity will provide us with a method for individuating objects in terms of their nuclear properties. The other axioms of the theory do not directly figure in the solutions to these problems, but they may largely be thought to play an auxiliary role, providing us with a fund of nuclear properties to be used in the application of the more central axioms.

Let us now present the internal criticisms. These stay within Parsons' basic approach to the extent that some form of the Link Hypothesis and of 
the Identity and Abstraction axioms is preserved. I have not always stated the arguments in full or set out the suggestions for improvement with complete formal precision. But it should be clear, from what I say, both how the arguments may be developed and how the suggestions may be worked up into a rigorously stated theory. To a large extent, the details may be gathered from the related discussion of the contextualist theory in [2].

\section{THE LINK}

I shall argue that if Parsons' theory is to be successful in individuating the objects from stories or other contexts, then the form of the Link Hypothesis needs to be modified.

There are two main problems. To appreciate the nature of the first, consider a story in which the nuclear properties directly attributed to two of its native characters are the same. The only apparent intra-story difference between them lies in their extra-nuclear properties: one is said to be admired, the other detested, one a better detective than Holmes, the other not, and so on. It would then appear that the two objects are distinct, since one has extra-nuclear properties in the story not possessed by the other. It would also appear that the objects have the same nuclear properties in the story. But it would then follow by the Link Hypothesis that they actually have the same nuclear properties and so, by the Identity Axiom, are the same.

Parsons is well aware of this difficulty and tries to get round it by arguing that just as existents have the nuclear weakenings of their extra-nuclear properties, so existents of stories have, within those stories, the nuclear weakenings of their extra-nuclear properties (cf. p. 198). It follows that the native objects of a story can be distinguished by these nuclear weakenings, if not by the explicitly given nuclear properties. One of the characters of our example will have the nuclear weakening of being admired, the other the nuclear weakening of being detested, and so on.

It may be conceded that normally such a move is justified. But what if the story is some kind of philosophical fantasy in which the rule of nuclear weakening is not assumed to have full reign? Perhaps it is an important part of the story that an object never has the nuclear weakening of its extranuclear properties. There would then appear to be no basis for attributing to an object the nuclear weakenings of the extra-nuclear properties attributed 
to it in the story; and so if two such objects differed in the story only in their extra-nuclear properties, they would be nuclearly indistinguishable.

Such an example suggests what we may dub the nuclear form of the Link Hypothesis:

A native object of a story has the nuclear weakening of an extranuclear property iff it has the extra-nuclear property in the story.

As it stands, this form of the principle says nothing about the nuclear properties of the object in the case in which they are not nuclear weakening. But this difficulty may be met, though this is not the only way, by assuming that each nuclear property is the nuclear weakening of some extra-nuclear property. ${ }^{5}$

With the adoption of the new form of the Link Hypothesis, the problem over the philosophical fantasy can be met; for even though the native objects may not have the nuclear weakenings of their extra-nuclear properties in the story, they will still have them in fact.

To appreciate the nature of the second difficulty, consider a story in which the only difference between two native characters $a$ and $b$ lies in the complex propositions attributed to them, those not directly of subjectpredicate form. Perhaps it is true in the story that $a$ is a cheat or is a liar $(C a \vee L a)$, but not that $b$ is a cheat or a liar $(C b \vee L b)$; and similarly for other complex propositions. Then $a$ and $b$ would appear to have the same properties in the story and hence, by either the original or nuclear form of the Link Hypothesis, have the same nuclear properties. Yet they would also appear to be distinct, in contradistinction to the identity axiom.

Presumably, in the face of such examples, Parsons would again resort to the strategy of 'going nuclear'. He would argue that since $a$ exists in the story and is a cheat or a liar, then he has the nuclear property of being a cheat or liar $\left(\lambda^{N} x(C x \vee L x)\right)$ in the story, while $b$ does not.

Such a move can be seen to depend upon a double intra-story inference: one from ' $a$ is a cheat or $a$ is a liar' ( $C a \vee L a)$ to ' $a$ has the extra-nuclear property of being a cheat or a liar' $(\lambda x(C x \vee L x) a)$, which is predicational in form; and the other from ' $a$ has the extra-nuclear property of being a cheat or a liar' $(\lambda x(C x \vee L x) a)$ to ' $a$ has the nuclear property of being a cheat or a liar' $\left(\lambda^{N} x(C x \vee L x) a\right)$, which is nuclear-predicational in form. As we have seen, the second of these intra-story inferences can be challenged. But so can the first. The story might be a philosophical fantasy in which it is shown that 
there are no complex properties or none that conform to $\lambda$-abstraction. There would then be no basis for supposing that a had in the story the complex extra-nuclear property of being a cheat or a liar, let alone its nuclear weakening.

To take care of such examples, it seems to me, we must take greater care over the sense in which an object can be said to have a property in a story. Parsons introduces the notion of plugging, but we need to distinguish two senses in which a property can be plugged up with an object. Given a property and an object, we may let the internally formed proposition be the result of plugging up the argument-place of the property with the object, and we may let the externally formed proposition be to the effect that the object has the property. Thus if the property is $\lambda x(C x \vee L x)$ and the object is $a$, then the internally formed proposition is that $C a \vee L a$, while the externally formed proposition is that $\lambda x(C x \vee L x) a .^{6}$ We may now say that an object implicitly has a property in a story if the internally formed proposition is true in the story, and that it explicitly has the property in the story if the externally formed proposition is true in the story.

Before we followed Parsons in taking the story-relative copula to have the explicit sense. We may now take it to have the implicit sense. Combining this change with the previous one then gives us the following form of the Link Hypothesis:

A native object of a story has the nuclear weakening of an extranuclear property iff it implicitly has the extra-nuclear property in the story.

This form of the hypothesis might be called the Indirect Link, in contrast to the original direct link form of the hypothesis.

We now see that our difficulties over the anti-platonic story are removed. For the object a will implicitly have the property $p=\lambda x(C x \vee L x)$ in the story, if not explicitly. It will therefore be distinguished from $b$ by its possession of the nuclear weakening $\boldsymbol{P}^{N}$ of $\boldsymbol{P}$.

It is worth noting that the encoder theorist will face the second of these two difficulties, but not the first. His naive formulation of the Link Hypothesis is that a native object encodes just those properties it has in its story. The first difficulty will not arise for him since it depends upon the distinc- 
tion between nuclear and extra-nuclear properties. However, the second difficulty will require him, in much the same way as the nuclear theorist, to distinguish between the implicit and the explicit copula.

Is there any way in which Parsons can avoid these modifications to the Link Hypothesis? It does not seem important to me that he should, though they do mar the simplicity of its original formulation. Perhaps the best he can say is that the nuclear properties that he needs really are available from the stories. Although the anti-nuclear story denies that its objects have the nuclear weakenings of their extra-nuclear properties, still it will be true in the story that they have those nuclear weakenings; and although the antiplatonic story denies that there are complex properties, still it will be true in the story that its objects have the complex properties.

The trouble with this proposal is that it makes the stories inconsistent; and yet there seems to be a clear difference between these stories and those that boast of such inconsistencies. Also, although the proposal might gain us distinctions in some cases, it would lose us distinctions in others. Imagine a story in which the crucial difference between two of its native characters consisted in the fact that one had the nuclear weakenings of its extra-nuclear properties, while for the other it was left open. If the second automatically acquired the nuclear weakenings within the story, then the distinction between the two objects would be lost.

Parsons seems to assume that his theory is internalized to stories, not in the sense of being part of them, but in the sense that they are closed under rules that it sanctions (cf. p. 200). He accepts not just the inference from an existent having an extra-nuclear property to its having the nuclear weakening, but also the inference from an existent within a story having an extra-nuclear property to its having the weakening within the story, and similarly for the rule of $\lambda$-abstraction. But we must be careful to distinguish between the external and internal validity of a theory. The rules of a theory of nonexistents are not so strong that they can penetrate to the wildest of all our fancies and dreams. They have a purely external validity, and govern the behaviour of the objects from outside of the contexts in which they appear, not from within.

But even if we confine our attention to stories that lie within the genre of realistic fiction, it is still not clear to me that the rules of nuclear weakening and $\lambda$-abstraction should have internal validity. The validity of these rules is, after all, a matter of philosophical controversy. As Parsons himself admits 
(p. 227), the existence of complex properties has often been doubted; and if the rule of nuclear weakening has been doubted less, it is only because it has not been considered more. Is it seriously to be supposed that these dubious pieces of philosophy are to be imported into the interpretation of fiction?

Yet conceding the internal validity of the rules, it is still possible to construct clear examples of realistic fiction that result in difficulties of the first kind (though, alas, not of the second). Consider a story with two native characters that not only fail to exist but fail to exist in the story (they are internal non-existents). Then might not the only difference between them in the story lie in their extra-nuclear properties? The usual technique of going nuclear would not help here. For given that the objects fail to exist in the story, we cannot reasonable infer that they have, in the story, the nuclear weakenings of their extra-nuclear properties.

Parsons tries to take care of this sort of problem (pp. 200-202). He suggests that there will be two main sources of information about the nuclear properties of such objects. There will be 'outside' information from the story itself. But also, the main story may place these objects within the context of a subsidiary story and so there will be 'inside' information about the objects from the subsidiary story itself. Given that it is part of the main story that the objects have certain nuclear properties in the subsidiary stories, they will have them in the main story and so have them in fact. The properties will pass from the subsidiary story, as it were, through to the main story, and out to the real world. From such information, suitably fleshed out non-existents of a story can then be distinguished.

There are various ways, though, in which such reasoning can fail us. It is not necessary that the main story should place the internal non-existents within the context of anything like a story. But let us grant that it does. Still, the nuclear properties of the objects in the subsidiary story will not be exportable unless they are taken in the main story to be native to their story. Without this assumption, the nuclear properties of the objects will be locked into the subsidiary story, and they can be as richly and variously described as one likes within that story without being distinguishable by their nuclear properties. (We might also note that exportation depends upon the Link Hypothesis being appropriately internalized to the main story; but we may grant this as part of the realistic constraints.) But ignoring this difficulty, it may be that the non-existents are indistinguishable within the subsidiary 
story, yet distinguishable through their extra-nuclear properties in the main story. Perhaps the difference between them lies in the roles they play in the lives of the main characters: one is admired, the other detested; one inspires noble deeds, the other no deeds at all. It would then be true in the main story that there was some nuclear property on which they differed, but there would be no specific nuclear property upon which they did differ. Or again, the difference between the two non-existents might lie in the fact that the one, unlike the other, was denied to have certain properties in the subsidiary story or said to have one property in the story if it has another. In all of these ways, and others too, there may fail to be a nuclear property that distinguishes the two internal non-existents.

So we see that the objections to our examples cannot be sustained and that the direct form of the Link Hypothesis must be abandoned.

But can we be sure that the Indirect Link is not also open to counterexample? It can, in fact, be shown that the Indirect Link is impervious to all criticism of the kind that we have been presenting. What must be shown is that objects distinguishable within their stories are distinguishable in terms of their nuclear properties. So suppose the objects $x$ and $y$ are distinguishable within their stories. This means that one, say $x$, will implicitly have in its story an extra-nuclear property $\boldsymbol{P}$ not had by the other in its story. By the Indirect Link, $x$ will have the nuclear weakening $\boldsymbol{P}^{N}$ of $\boldsymbol{P}$. But granted that $P^{N}$ is not the nuclear weakening $Q^{N}$ of any other property $Q$, it follows that $y$ does not have the nuclear property $\boldsymbol{P}^{N}$. So, in case the Indirect Link Hypothesis is adopted, we shall actually have a proof that story distinguishable objects are nuclearly distinguishable.

There are, however, certain peculiarities in the application of the Indirect Link which, though not damaging, are worth pointing out. First, it follows that not all objects are possible objects of thought, in the sense that there can be a story or other context to which they are native. For consider the object $a$ whose sole nuclear property is the property $\lambda^{N} x(x=x)$ of being self-identical. Then $a$ cannot be native to any context. For if it were, the proposition that $a=a$ would be true in that context. So $a$ would implicitly have the property $\lambda x(x=a)$ of being identical to $a$ in that context. And therefore it would also have the nuclear property $\lambda^{N} x(x=a)$ of being identical to $a$. This result implies, however, no essential limitation on our powers of conception. It is merely a consequence of the fact that an object cannot implicitly have certain properties in a story without implicitly having others. But it may 
still be true that each abstract content, as given by a cluster of properties or relations, can appropriately be realised in thought.

Second, the satisfactory individuation of objects may depend both upon iterating the operation $\lambda^{N} x$ of nuclear abstraction and upon supposing that the iterations produce new properties. A good example of this arises from consideration of the existent golden mountain. This object, call it $e_{1}$, will be individuated in terms of the nuclear properties of being existent, golden and a mountain. (There may be other nuclear properties, but only the nuclear property of existence will concern us.) Let us use $E_{0}$ for the extra-nuclear property of existence and $E_{1}$ for the nuclear property $\lambda^{N} x E_{0} x$ of existence. Consider now the existent ${ }_{1}$ golden mountain, $e_{2}$, and let $E_{2}$ be the nuclear property $\lambda x^{N} E_{1} x$ of existence $e_{1}$. The question arises as to whether $e_{1}=e_{2}$ and $E_{1}=E_{2}$. Now $E_{0} \neq E_{1}$, since $e_{1}$, the existent golden mountain, exists but does not exist. It then seems reasonable that $e_{1} \neq e_{2}$, since one is characterised as existent $t_{0}$ and the other as existent ${ }_{1}$. But then the only way of distinguishing between $e_{1}$ and $e_{2}$ is in terms of $E_{1}$ and $E_{2}$. It therefore follows that $E_{1} \neq E_{2}$. Proceeding in this way, we see that all of the iterations $E_{0}, E_{1}$, $E_{2}, \ldots$ and all of the corresponding objects $e_{0}, e_{1}, e_{2}, \ldots$ are distinct from one another.

Finally, the Indirect Link no longer gives us a direct basis for the attribution of nuclear properties to non existents. A proposition of the form $\mathrm{Pa}$ will rarely, if ever, be true because that same proposition is true in the story to which the object $a$ is native. What the truth of $P a$ in the story most directly justifies is the proposition $\left(\lambda^{N} x P x\right) a$; and so it is only if the property $\lambda^{N} x P x$ should turn out to be identical to $P$, that the proposition $P a$ will also be justified.

This is in striking contrast to the Direct Link. For there what justifies $P a$ is always the truth of that very same proposition in the story. However, as far as I can see, this feature does not impair the ability of the Indirect Link to explain the truth of literalist judgements, such as 'Holmes is a detective' or 'Hamlet is a prince'. For suppose that being a detective is the complex nuclear property $\lambda^{N} x \varphi(x)$. Then it will be true in the Conan Doyle stories not just that $\lambda^{N} x \varphi(x)$ of $a$, but also that $\varphi(a)$; and so, by the Indirect Link, Holmes will have the nuclear property $\lambda^{N} x \varphi(x)$. The Direct and Indirect Links will predict slightly different literalist truths for certain special stories; but on these, our intuitions will not, in any case, be clear.

It should be noted, though, that the original form of the Link Hypothesis 
can be retained as long as our sole concern is to explain the truth of literalist judgements. The modifications to the hypothesis only arose from the further demand that objects be individuable in terms of their nuclear properties.

\section{CORRELATES}

Two objects are said to be correlates if the nuclear properties of either one involve the other. For example, the Watson and Holmes of Conan Doyle's stories are correlates, since Watson will have the nuclear property of living with Holmes and Holmes the nuclear property of living with Watson.

Stories and other contexts naturally give rise to correlates. For suppose some proposition $\varphi(a, b)$ involving the native objects $a$ and $b$ is true in a story. Then by the Indirect Link Hypothesis, $a$ will have the property $\lambda^{N} x \varphi(x, b)$ and $b$ will have the property $\lambda^{N} y \varphi(a, y)$; and so $a$ and $b$ will be correlates. The Direct Link will also readily give rise to correlates. If, for example, the proposition that $a R b$ is true in the story, then, by Parsons' theory of relations or realistic intra-story inference, $a$ will have $[R b]$ and $b[a R]$.

The presence of correlates gives rise to difficulties, both for the problem of ontology and for the problem of identity. In fact, the difficulties over correlates are an extreme case of a more general difficulty, that arises whenever the individuation of non-existents leads in a circle, i.e. whenever the non-existents involved in the nuclear properties of one non-existent lead through the nonexistents involved in their nuclear properties, and so on back to the original object.

In the case of the problem of ontology, the difficulty is that the theory of objects, as set out by Parsons, is incapable of proving the 'existence' of correlates. It is impossible to prove, for example, that there is a nuclear relation $R$ and objects $x$ and $y$ such that $[R y]$ is the sole nuclear property to be possessed by $x$ and $[x R]$ is the sole nuclear property to be possessed by $y ;^{7}$ and this example is but one out of many.

Parsons (pp. 194-197) is aware of this difficulty; but his response is curious. He seems to suggest that such principles should be obtained by combining the theory "with its application to fictional objects". Presumably what he has in mind is that we find in fiction the native objects with the correlative nuclear properties and then use the Link Hypothesis to establish that there are objects with those properties. But by the same token, one 
might as well dispense with the abstraction axiom for objects and let the application of the theory determine that there are the objects that would otherwise be determined with its help. Surely we want the theory to explain how there can be the objects required by fiction, not to presuppose that there are such objects. The theory should anticipate what objects are to be found in fiction, not help itself to them.

If the difficulty over correlates is to be met, then the axioms of the theory must be supplemented. But how? One possibility, considered but not endorsed by Parsons (pp. 196-197), is that the axioms for the identity of properties are to blame. We may suppose that all nuclear properties $[R x]$ or $[x R]$, for all non-existent $x$, are the same. There is then no problem in proving the existence of correlates, since a single property $P$ could be used in place of their correlative properties.

But surely the assumption underlying this proposal is false. The property of living with Holmes just is not the same as the property of living with Hamlet; the Conan Doyle stories ascribe the one to Watson, but not the other (cf. p. 197). But even if we take a more formalistic approach to property theory and only individuate properties to the extent that they are required to individuate objects, difficulties will remain. One counter-example is considered by Parsons on pp. 196-197. Another, especially troublesome, case is that of two stories that differ only in the substitution of one immigrant character for another. If the two immigrant characters are sufficiently alike at source, then the native characters of either story would appear to be the same.

We may further note that the proposal requires not just the identification of all relational properties of the form $[R x]$ and $[x R]$, for $x$ non-existent, but also the identification of all nuclear properties involving non-existents. But this is not something that can consistently be maintained with the rest of the theory. To take an artificial example, $\lambda^{N} x\left(x=a_{1} \wedge b=b\right)$ must be distinct from $\lambda^{N} x\left(x=a_{2} \wedge b=b\right)$, for $a_{1}$ and $a_{2}$ distinct existents and $b$ a non existent, since $a_{1}$ has the one property but not the other.

It must be accepted that the correct solution to this problem is the most obvious one: the abstraction axiom for objects must somehow be supplemented. But how should the new formulation go? Once the great variety of correlative objects, as abstractly considered, is taken in, it will be appreciated that there is no simple way to 'fix' the axiom. Let me merely state how I 
think the new formulation should go without attempting to retrace the steps by which it was reached. These steps may to some extent be recovered from the discussion of the related problem in Section D4 of [2].

Call a proposition nuclear if it is of the form $R a_{1} \ldots a_{n}$ for $R$ a nuclear relation or property. Let the nuclear content of the objects $a_{1}, \ldots, a_{n}$ consist of all the nuclear propositions in which at least one of $a_{1}, \ldots, a_{n}$ figures as a subject. (In the more general case, we would allow for infinitely many objects here and even for infinitary relations.) Call an $n$-ary extra-nuclear relation $\boldsymbol{R}$ quasi-nuclear if the implicity formed proposition resulting from plugging the $n$ objects $a_{1}, \ldots, a_{n}$ into $R$ is always a nuclear proposition involving at least one of $a_{1}, \ldots, a_{n}$ as subjects. The relations $\lambda x y(x[R y])$ and $\lambda x y\left(\lambda^{N} z \varphi(z, x) y\right)$, for example, are quasi-nuclear. Now say that a class $C$ of quasi-nuclear $n$-ary relations is the abstract nuclear content of the objects $a_{1}, \ldots, a_{n}$ if (i) all of $a_{1}, \ldots, a_{n}$ are distinct, (ii) no relation of $C$ involves any of $a_{1}, \ldots, a_{n}$, and (iii) the nuclear content of $a_{1}, \ldots, a_{n}$ is obtained by plugging the objects $a_{1}, \ldots, a_{n}$ into the relations of $C$.

Then the revised and expanded version of object abstraction becomes:

Any class $C$ of $n$-ary quasi-nuclear relations is the abstract nuclear content of some objects $a_{1}, \ldots, a_{n}$.

As it stands, this axiom is not quite right. For letting $C=\left\{\lambda x_{1} x_{2} P x_{1}\right.$, $\left.\lambda x_{1} x_{2} P x_{2}\right\}$, it follows that there are two distinct objects whose sole nuclear property is $P$, in contradistinction to the identity axiom. To rule out such cases, we must require that there not be any symmetry among the argumentplaces of $C$ (a notion that may be made precise in terms of the relations induced through a permutation in the argument-places). Such a restriction is awkward and somewhat mars the simplicity of the scheme, but it has a rather satisfactory consequence in case $C$ is empty. It will then follow that $n=1$; for otherwise there would be a degenerate symmetry among the argument-places of the content. Thus there will be a null object, but no 'null correlates'.

The revised axiom also does not explicitly allow for the possibility that existents may be subjects of nuclear propositions involving non-existents. This is all to the good if one does not believe in such a possibility. But otherwise it is necessary to further complicate the formulation of the axiom.

We can think of the revised version of abstraction in the following way. Given any distinct objects $a_{1}, \ldots, a_{n}$, we can form their nuclear content; 
and from this, by abstraction from their occurrence in the nuclear propositions, we can form their abstract nuclear content. The new axiom then says that, conversely, any appropriate class of relations can be obtained as the abstract nuclear content of some objects.

To see how the axiom works in a particular case, consider characters Watson and Holmes from an artificially thin story, whose nuclear content consists of the propositions that Watson is a medical doctor, that Holmes is a detective, and that Watson kicked Holmes ( $\S M w, \S D h$ and $\S w[K h])$. (Nothing turns on the artificiality of the example.) Let $C$ consist of the following quasi-nuclear relations: $\lambda x_{1} \dot{x_{2}} M x_{1}, \lambda x_{1} x_{2} D x_{2}$ and $\lambda x_{1} x_{2}\left(x_{1}\left[K x_{2}\right]\right)$. Then the new axiom gives us distinct objects $a_{1}$ and $a_{2}$ for which $\left\{\S M a_{1}\right.$, $\left.\S D a_{2}, \S a_{1}\left[K a_{2}\right]\right\}$ is their nuclear content. Or again, the axiom will give us distinct objects, call them the Small and the Large, such that the sole nuclear proposition true of them is that the Small is smaller than the Large. (This may help with Plato's theory of forms.)

As the new axiom has been stated, it is not formulable within the resources of Parsons' theory. This can be done by introducing a new primitive, with the sense that an object 'occurs' or is 'involved' in a proposition. ${ }^{8}$ But in that case, the plugging operator would need to be given the internalist interpretation and further axioms concerning the identity of intensional entities would need to be added to the system for any reasonable deductive development. To some extent, a generalized form of abstraction can be stated without using the notion of involvement or presupposing a structural conception of relations or propositions. But such a formulation, in so far as it was adequate, would be very complicated; and so I shall not go into details.

Let us now turn to the difficulties correlates raise for the problem of identity. Consider again an artificially thin story, one in which the sole proposition is that Watson kicked Holmes. It then seems reasonable that there should not be another story which differed from the original story only in its native objects. If the content of that story was provided by the proposition that Watson' kicked Holmes', then Watson' should be identical to Watson and Holmes' to Holmes. Call two objects $a$ and $b$ strict $R$-correlates if they are distinct and if the sole nuclear property of $a$ is $[R b]$ and the sole nuclear property of $b$ is $[a R]$. Then what one requires is that if the pair (Holmes, Watson) are strict $K$-correlates, for $K$ the relation of kicking, then there should not be any other pair of objects that are also strict $K$-correlates. More generally, what is required is that if a sequence of objects is correlated 
through their nuclear properties or relations in a certain way, then there should not be any other sequence of objects that is so correlated.

What makes this requirement so reasonable is the demand that the theory be capable of individuating non-existent objects in a non-circular way. If two pairs of objects $(a, b)$ and $(c, d)$ could both be strict $R$-correlates, we would have no way of identifying both members of a pair without already presupposing the identity of one of them. We could say what $a$ was given $b$, and what $b$ was given $a$, but not what $a$ and $b$ were independently of either. On the other hand, if $(a, b)$ were the only pair of objects to be strict $R$ correlates, then they could simultaneously be identified, without circularity, as the unique pair of objects $(x, y)$ for which $[R y]$ was the sole nuclear property of $x$ and $[R x]$ the sole nuclear property of $y$.

Even if the demand for non-circular identification were given up, the uniqueness assumptions are required to avoid embarrassing metaphysical questions. If there can be distinct pairs of correlates, then how many of them can there be? If the Link Hypothesis leads to two native objects of a story being correlates, how do we tell, if there are several such pairs of correlates, which particular pair they are? These questions seem to be 'metaphysical' in the bad sense of the term; they have no answer only because they should not have arisen.

Parsons does not explicitly discuss non-circular individuation, but there is textual evidence that he wants it. On p. 185, he writes: "On the theory given here, if a piece of fiction accidentally turned out to be a complete and completely accurate account of the entire (past, present and future) history of the universe, then all characters created therein would be identical with real objects." And on p. 188 he writes: "It is a consequence of my views that if two authors were to write exactly the same story, they would thereby have created the same characters." But neither thesis is a consequence of his theory as it stands. If, for example, the characters of the two stories were already different, then an application of Object Identity would not force them to be the same, since their correlative nuclear properties would also be distinct. The endorsement of either thesis would appear to arise from a commitment to non-circular individuation.

The rationale for the identity axiom, though not its content, would also seem to involve such a commitment. At various places throughout the book, Parsons is concerned to defend the identity axiom against counter-examples. Now none of the counter-examples is a logical fantasy in which distinct 
objects are said to be the same or in which native objects are not said to be identical to themselves. So why did he not, instead of constructing the elaborate defences that he does, simply identify each native object $a$ in terms of the nuclear property of being identical to $a$ ? Surely the answer is that he is concerned to provide, not any distinguishing nuclear property, but one that distinguishes the object without circularity.

In this connection, the discussion on p. 200 is especially revealing. He is there concerned to distinguish between an existent and imaginary policeman within a story. He suggests that the existent policeman has the nuclear property of being distinct from the imaginary policeman, while the imaginary policeman does not. But why did he not use the simpler nuclear property of being identical to the existent policeman? Presumably, his thought is that the imaginary policeman can be identified independently of the existent policeman and that in this way, unlike the other, the existent policeman can then be identified without circularity in terms of the non-existent policeman.

It seems, then, both in regard to what is reasonable and what Parsons wants to say, that the theory should provide for the non-circular individuation of non-existents. But the theory, as it stands, is incapable of doing this. It is incapable, for example, of proving that pairs of strict $R$-correlates are the same. ${ }^{9}$

As before, it is possible to locate the fault in the axioms for the identity of properties; but there are again the same objections to this proposal. It seems better to extend the identity axiom for objects. Taking into account the whole gamut of correlative objects, what we need to say is that the abstract nuclear content of any $n$ distinct objects uniquely determines those objects. That is:

If two $n$-tples $x_{1}, \ldots, x_{n}$ and $y_{1}, \ldots, y_{n}$ have the same abstract nuclear content, then the $n$-tples are the same, i.e. $x_{1}=y_{1}, \ldots$, $x_{n}=y_{n}$.

The new axioms of abstraction and identity effect a fundamental shift in the perspective on the objects of the theory. For Parsons, objects are determined singly, in terms of their nuclear properties. For us they are determined simultaneously in terms of their nuclear properties and relations. One can, if one likes, think of nuclear predications as containing variables for "unknown objects". Then for Parsons, one can uniquely solve for any suitable class of 
equations in a single unknown; while for us, one can uniquely solve for any number of unknowns.

The more limited perspective of Parsons may arise from a false analogy with the theory of properties or relations. Properties and relations are singly determined by the appropriate forms of abstraction; and therefore it is supposed that the objects must also be singly determined. But a better understanding of the analogy between the two types of abstraction leads to a different view. In the objectual analogue of an abstraction axiom, the role of subject and predicate is reversed. Thus corresponding to the principle that every condition on objects determines a property $(\exists P \forall x(P x \equiv \varphi(x)))$ is the principle that every condition on properties determines an object $(\exists x \forall P(P x \equiv \varphi(P)))$. If now we start with the relational form of abstraction, that every condition on pairs of objects determines a relation $(\exists R \forall$ $x \forall y(R x y \equiv \varphi(x, y)))$, the reversal of roles gives us the principle that every condition on relations determines - not an object, since that makes no sense - but a pair of objects. The multiplicity of subject terms in a relational statement forces upon us the simultaneous determination of the objects in the corresponding axiom of abstraction.

The new perspective, once adopted, brings several advantages in its train. It fits in well, first of all, with a generative or constructive conception. According to such a conception, the objects, properties and relations are generated in a definite order, much as are the sets of the cumulative hierarchy. Thus properties and relations help to generate objects; these objects help to generate new properties and relations; and so on.

Now as Parsons himself points out (pp. 195-196), it is difficult for him to maintain such a generative conception. For on his view, objects must be generated from properties. So Holmes must be generated with the help of the property of living with Watson, which must be generated with the help of Watson. But in the same way, Watson must be generated with the help of Holmes; and so we are left with a circle.

On our view, the difficulty disappears. Objects will be generated simultaneously from abstract nuclear contents. We might, in reductive fashion, think of them as ordered pairs, consisting of a nuclear content and an index to indicate which argument-place the object is to fill. Thus the native characters of the Conan Doyle stories will emerge together from the appropriate abstract nuclear content. In such a way, the circle that arises from correlates will be avoided. 
Another advantage of the enlarged conception of objects is that it avoids the complications in Parsons' theory of relations. Parsons' theory is motivated by two main concerns. The first, and more important, is to give some account of when relational statements between non-existents hold (cf. p. 67). The theory does give some account of when subject-predicate statements $P a$ hold, for it will be part of the 'identity' or 'definition' of the non-existent $a$ that it should have the property $P$; but it fails to give a similar account of when relational statements $R a b$ hold. The second, and less important, concern is to uphold the intuition, deriving from Bolzano, that no existent enter into a nuclear relation with a non existent.

Parsons solves the first of these problems with his stipulation that the relational statement $R a b$ should be defined by the conjunction of $a[R b]$ and $[a R] b$. The truth of the relational statement will then be reduced to the attribution of nuclear properties, which will flow from the definitions of the objects in the normal way. The second of the problems is solved through his assumption that no existent have a relational property $[R x]$ or $[x R]$ involving the non-existent $x$.

But there is, in this theory, a significant ambiguity. For as in the case of properties, the notion of plugging for relations may be given either an internal or an external sense. If $R$ is the relation $\lambda^{N} x y \varphi(x, y)$, then the external plugging of $a$ in the first argument-place will be $\lambda^{N} y a R y\left(=\lambda^{N} y\right.$ $\left.\left(\left[\lambda^{N} x y \varphi(x, y)\right] a y\right)\right)$, while the internal plugging up will be $\lambda^{N} y \varphi(a, y)$. Even though he has the notation for nuclear abstraction, Parsons nowhere says whether plugging is to be taken in the internal or external sense or in some other sense altogether.

If plugging is taken in the external sense, then a significant simplification can be effected in his theory. Relational statements may be reinstated as part of the primitive notation of the theory and plugs, such as $[x R]$, may be dropped as primitive and be defined instead in terms of abstracts, such as $\lambda^{N} y(x R y)$. In this way, the complicated axioms for plugs may be derived from the usual axioms for $\lambda$-abstraction; and we shall be left with Parsons' definition of relational statements as an axiom:

(*) $\quad x R y \equiv[x R]$ y $\wedge[R x]$,

along with the axiom forbidding intercourse of the existent with the nonexistent.

Parsons himself briefly considers a similar proposal, but rejects it on the 
grounds that there is then no adequate account of the relational statement $x R y$ (fn. 3, p. 67). But here there is an error. Given a relation $R$, we can understand the identity of $\lambda^{N} y x R y$ or $\lambda^{N} x x R y$ apart from their application conditions as well as we can understand the identity of $[x R]$ or $[R y]$. (This is particularly clear on a structural conception of properties, since then their identity is given by their relevant structure and is completely independent of their conditions of application.) Now to define the objects we do not need the application conditions of the properties. So the properties $\lambda^{N} y(x R y)$ and $\lambda^{N} x(x R y)$ may be used to help define new objects and the axiom (*) then used to determine the application conditions of $R$. In other words, we may make a route from the identity of $R$, to the identity of $\lambda^{N} y(x R y)$ and $\lambda^{N} x(x R y)$, through to the identity of objects with those properties, and so to the application conditions of $R$. The mystery of relational statements is solved as well as it is on Parsons' account.

However, the whole idea that there is a special problem about relational statements is not one that should arise. It only arises for Parsons because of his unduly narrow conception of how objects are defined. Since they are only given in terms of their nuclear properties, the basis for relational attributions does indeed appear problematic. But take objects to be simultaneously defined in terms of their nuclear properties and relations, and the problem disappears. The nuclear relations into which an object enters will be given in the same direct way as the nuclear properties which it possesses. It will be as much a part of the definition of Holmes and Watson that the one lives with the other as that one is a detective and the other a doctor.

But although the problem that motivated $(*)$ as a definition disappears, the question of the truth of $(*)$ as a thesis remains. Now on the external interpretation of plugging, the thesis is not at all plausible; and so although the formulation of the axioms can be simplified under this interpretation, it is not a simplification that I would recommend. On the other hand, under the internal interpretation of plugging - and this came as a great surprise to me - it may plausibly be argued that the thesis is true, at least in its application to those objects that are real or are native to a story or other context.

For take $R$ to be of the form $\lambda^{N} x y \varphi(x, y)$. Suppose that $a R b$. If $a$ and $b$ both exist, then it is clear that $a[R b]$ and $[a R] b$. Given that existents do not enter into nuclear relations with non-existents, the only other case is that $a$ and $b$ are both non existent. Now it is plausible to extend one direction of the Link Hypothesis to relations in the following way: 
If $x$ is native to a story s, then $x R y$ only if $x$ and $y$ implicitly have $R$ in the story s.

Since $a$ is non-existent, it is native to some story $s$, say. By the above conditional, $a$ and $b$ implicitly have $R$ in $s$. So the proposition that $\varphi(a, b)$ is true in s. So $a$ implicitly has $\lambda x \varphi(x, b)$ in $s$. But $[R b]$ is $\lambda^{N} x \varphi(x, b)$ and therefore, by the non-relational form of the Link Hypothesis, $a[R b]$. In the same way, it may be shown that $[a R] b$. Now suppose $[a R] b \wedge a[R b]$. If $a$ and $b$ both exist, it is clear that $a R b$. Granted that existents cannot have nuclear properties $[x R]$ or $[R x]$, for $x$ non-existent, the only other case is that $a$ and $b$ are both non-existent. The objects $a$ and $b$ are then native to respective stories $s$ and $t$, let us say. By the usual form of the Link Hypothesis, the proposition that $\varphi(a, b)$ is true in both $s$ and $t$. Suppose that $s$ and $t$ are distinct. Then given that an object is native to at most one story, it follows that there are two stories $s$ and $t$, with $a$ native to $s$ but immigrant to $t$ and $b$ native to $t$ but immigrant to $s$. But this situation may be ruled out as ill-founded. So we may assume that $s$ and $t$ are identical. Extending the other direction of the Link Hypothesis to relations, we have:

The objects $x$ and $y$ bear $R$ if they are native to the same story and implicitly bear the relation $R$ in that story.

Since $a$ and $b$ are native to the same story and implicitly have $R=\lambda x y \varphi(x, y)$ in that story, it follows that $a R b$.

The above derivation depends upon certain assumptions, but they are all faily plausible. We may also note that, conversely, the relational form of the Link Hypothesis may be derived as long as (*) is adopted as an assumption. So we see that although the original motivation for the thesis - the elimination of relational statements - was misconceived, it may still be retained for the related purpose of eliminating the relational applications of the Link Hypothesis.

\section{THE PARADOXES}

Since object theory adopts an abstraction principle for objects, it is not surprising that it falls foul of Russell-type paradoxes. There are basically two kinds of paradox that can arise, one deriving from the lack of restriction on properties, and the other from the lack of restriction on objects. I shall 
here discuss how these paradoxes arise and how they are to be resolved in the context of Parsons' theory. Similar paradoxes have been discussed elsewhere; and the reader may consult [2], D2-3, for a fuller account and further references.

To see how the first paradox arises, consider again the principle of property abstraction:

For any condition there is a property that an object has iff it satisfies the condition.

From this principle, it follows that:

(†) There is no way of correlating each property with an object (with distinct properties correlated to distinct objects).

The proof is straightforward, but we can see intuitively why the consequence should hold through considerations of cardinality. For if there were the proposed correlation, there would be at least as many objects as properties; but by property abstraction, it follows that there must be more properties than objects.

Now in an object theory, some form of object abstraction is likely to be in conflict with ( $\dagger$ ). In Parsons' theory, the conflict arises in the following way. For any property $\boldsymbol{P}$, there will be a nuclear weakening $P$. With each such nuclear weakening may be associated an object $x$ for which $P$ is the sole nuclear property. But given what we may call the Distinctness Thesis:

Nuclear weakenings of distinct extra-nuclear properties are distinct $(\boldsymbol{P} \neq Q$ implies $P \neq Q)$,

the above procedure then correlates each property $\boldsymbol{P}$ with a distinct object $x$.

Parsons' solution to this paradox is, in effect, to drop the Distinctness Thesis (fn. 14, pp. 203-204). But this will not do. First, there is the danger that the theory will not properly be able to distinguish distinct objects. Suppose $\boldsymbol{P}$ and $Q$ are distinct extra-nuclear properties for which $P=Q$. Perhaps the only difference between two objects is that to one is attributed $P$ in its native story, while to the other is attributed $Q$. There would then be no basis for distinguishing between them in terms of their nuclear properties. Parsons mentions this difficulty, but suggests that the thesis "could probably be maintained for typical cases of extra-nuclear predications in stories" (pp. 203-204). But the difficulty needs to be met not just for the typical 
but for the hypothetical cases as well; and it may then be plausibly argued that there is no reasonable class of properties for which the thesis holds. For suppose there were such a class. Then one would like it to contain the property of being an object that lacks any property $P$ of the class for which $P$ is its sole nuclear property. But this is enough to regenerate the paradox.

A more serious objection to dropping the thesis is that it leaves us with no definite conception of what nuclear weakening is. With the thesis, we can think of the identity of the nuclear weakenings as being parallel to or even derivative from the identity of their extra-nuclear counterparts. If, on a structural conception of properties for example, $P$ is the property $\lambda x \varphi(x)$, then we can conceive of the weakening $P=\lambda^{N} x \varphi(x)$ as being just like $P$, but with the nuclear operation of abstraction in place of the extra-nuclear operation. Without the thesis, however, we are left completely in the dark as to the identity of nuclear weakenings; and nor does there seem to be any sensible and non-circular account of what it might be.

My own favoured solution to the paradox is to drop the principle of property abstraction. There still will be, for any condition $\varphi(x)$, the property $\lambda x \varphi(x)$ of being a $\varphi$-er; but this property, in line with the condition $\varphi(x)$, may be neither true nor false of certain objects and so may fail to conform to the bivalent interpretation of the abstraction principle. The details of such a proposal comprise a large and difficult question. But we may note that some such proposal is probably required, in any case, to deal with the classical paradoxes; and so the present solution may be seen, not as an ad hoc suggestion, but as part of a more general strategy for dealing with the paradoxes.

The second paradox in object theory arises from the principle of object abstraction. In Parsons' theory, this principle takes the form:

For any condition $\varphi$ on nuclear properties, there is an object that has a nuclear property iff it satisfies the condition.

From this principle, it follows that:

There is no way of correlating each object with a distinct nuclear property.

Again, we can see intuitively why this consequence should hold; for if there were the correlation, there would be at least as many nuclear properties as objects, whereas, by the abstraction principle, there must be more objects than properties. 
Now the principles concerning the identity of properties are likely to run into conflict with this consequence. For take any nuclear relation $R$, the nuclear relation of identity, say. It seems natural to assume that $[R x]$ and $[R y]$ are distinct for distinct $x$ and $y$. But then the association of $x$ with $[R x]$ gives the desired correlation of objects with nuclear properties (p. 235).

It is to be noted that a similar difficulty arises when any term of the form $\lambda^{N} y \varphi(x, y)$ is used in place of $[R x]$; and so the problem is not one that concerns the notion of plugging alone. In case $\varphi(x, y)$ is the formula ' $x=y$ ', an independent argument may be used to establish the distinctness claim. For the nuclear properties $\lambda y(x=y)$ must be different for different $x$, since only $x$ has $\lambda y(x=y)$. But then granted then Distinctness Thesis, the nuclear properties $\lambda^{N} y(x=y)$ will be different for different $y$.

Parsons considers two solutions to this paradox. The first is to give up the principle that $x \neq y \supset[R x] \neq[R y]$. But is it not possible to believe of an object that it has $[R x]$, but not believe that it has $[R y]$ ? Only, suggests Parsons, when $x$ and $y$ are conceivable objects, ones that can be had in mind. So the above principle need not be retained in its full generality, but only in its application to the conceivable objects (p. 236).

However, this solution will not do. As in the case of the first paradox, there would appear to be no reasonable class of objects for which the disputed principle held. To mimic the previous argumentation, suppose that $x \neq y$ entailed $[R x] \neq[R y]$ for all conceivable $x$ and $y$. Consider now the object $x$ whose nuclear properties are just those $P$ for which there is a conceivable $y$ with $P=[R y]$ and $\sim P y$. Presumably, $x$ itself is a conceivable object. But then the paradox returns in full force. ${ }^{10}$

There is, however, a more serious objection to giving up the assumption that $x \neq y$ implies $[R x] \neq[R y]$; for as with the Distinctness Thesis, we are then left with no definite or reasonable conception of what the properties $[R x]$ are like. If $[R x]$ and $[R y]$ can sometimes be the same, for distinct $x$ and $y$, and sometimes distinct, how is it to be decided which? Indeed, the problem is even worse than this question might suggest; for if paradox is to be avoided, it is not only required that $[R x]$ and $[R y]$ be the same for some distinct $x$ and $y$, but also that some such $x$ and $y$ differ in whether they possess the property $[R x]$. But if $R$ is nuclear identity, let us say, how is it to be decided, given that $[R x]$ and $[R y]$ are the same, whether it is $x$ or $y$ that is to have the property? There seems to be no definite or reasonable way of resolving such questions. 
Parsons' other proposal is to amend the abstraction axiom for objects. He suggests that a condition on properties only be taken to determine an object when its object quantifiers are restricted to existents. This proposal is somewhat reminiscent of Russell's ramified theory, with the existents being of order 0 and the non-existents of order 1 .

Now certainly the system, as thus amended, is consistent (thereby answering the first of the problems on p. 240). But it is unclear, first of all, what general conception of objects underlies the amended system. It is not strict ramification in the sense of Russell, since the relations used to define the objects in the revised axiom may themselves be defined in terms of unrestricted quantification over objects. Indeed, it is a kind of freak that the amended system is consistent at all. Suppose extra-nuclear predicates of nuclear properties were introduced into the system, with an appropriate form of abstraction. Then inconsistency would be restored.

But even if this difficulty is put aside, it is still not clear that the revised system is adequate for its intended applications. If it is only required that non-existents with finitely many nuclear properties be defined, there is no need for either restricted or unrestricted quantifiers; for object abstraction can simply take the form:

$$
\forall P_{1} \ldots \forall P_{n} \quad \exists x \forall P\left(P x \equiv P_{1} x \vee \ldots \vee P_{n} x\right) .
$$

But there seems to be something arbitrary about restricting the principle in this way; and, in any case, the existence of stories closed under a reasonable amount of inference would sometimes seem to require that their objects have infinitely many nuclear properties. In such cases, it is hard to see how to dispense altogether with the use of unrestricted object quantifiers in the formulation of the appropriate condition on the properties. We may wish to say, for example, that an object has all of the nuclear weakenings of the extra-nuclear properties implied in some sense by the extra-nuclear properties $\boldsymbol{P}$ attributed to it in its story. The question then arises as to how the relevant notion of implication is to be defined. One natural definition is that it be necessary, for all objects $x$, that if $\boldsymbol{P}$ of $x$, for every property $\boldsymbol{P}$ in the given class, then $Q$ of $x$. But an existentially restricted quantifier could hardly be used in place of the unrestricted quantifier; since then the class of consequent properties $Q$ would be too large. And nor it is clear in what other way the unrestricted quantifier could be dispensed with.

One perhaps detects, in these proposed solutions to the paradox, an overly 
formalistic attitude towards the task of constructing an adequate theory of objects. As in the early days of set theory, it is as if it were sufficient to construct a system that was strong enough to yield all theorems required for the application, but not so strong as to be inconsistent. Yet it should go without saying that we are after, not merely a formal solution to the paradoxes, but also a reasonable conception of what it is that we might have been talking about in the first place. ${ }^{11}$

My own favoured solution to the paradox is to amend the abstraction axiom for objects, though not in the way required by Parsons. I wish to say, instead, that every set of nuclear properties determines an object, but not necessarily every condition or class (and similarly for the relational forms of abstraction). The consequence of the paradoxical argument will now be that not every condition on nuclear properties determines a set of those properties - which is what one would already expect.

Such a view fits in well with the cumulative conception of objects. We can think of the objects, properties and relations as being generated in succession, with the nuclear properties and relations at any one stage being used to define new objects, and with the objects at any one stage being used to determine new properties and relations, not as members of their extension but as constituents in their defining condition. At no stage of the constructive process will anything more than a set of properties, relations or objects be generated. So it is only sets of properties or relations that will define objects and only sets of objects that will figure in properties.

Although this view is so natural, it is not one that Parsons considers. ${ }^{12}$ There are perhaps various reasons for this. One is that the view is not readily formulated within the symbolism of his system. (There may be a lesson to be learned here on the dangers of premature formalisation.) Another reason is that Parsons may already have felt obliged to abandon the cumulative conception of objects in the light of the difficulties over correlates. Thus an inadequate view on one problem may have lead to an inadequate solution of another.

\section{ESSENTIALISM}

Parsons briefly discusses the extension of his theory to a modal language (pp. 98-103, 186-187, 224-226), and I here wish to take him up on two of the issues that he raises. I should emphasize, though, that I am more concerned 
with how well different modal claims fit in with his general conception of objects than with the absolute truth of those claims.

The first question concerns the rigidity of nuclear attributions to nonexistents. Does a non-xistent necessarily have the same nuclear properties when it does not exist $\left(\forall x \forall P((-E x \wedge P x) \supset \square(-E x \supset P x)) ?^{13}\right.$ One might call this an essentialist question; though it is not one of the usual sort since, in it, non-existence plays the role that is usually played by existence.

The rigidity assumption is very natural on Parsons' conception of objects; for one is inclined to say that the nuclear properties of an object determine its identity as a non-existent, not merely in the weak sense of distinguishing it from every other object, but also in the strong sense of being constitutive of the object, of being essential to it as a nonexistent.

However, this is a question upon which Parsons is prepared to remain neutral. He is content to return to a former concern of his and merely to discuss the technical possibilities for maintaining some form of anti-essentialism within his formal theory (pp. 224-226). But is this satisfactory? The question of the identity of non-existents in the strong or modal sense of that term really is of great importance. Nor is it clear that there is any viable alternative to the rigidity assumption. If the nuclear properties of a non-existent can vary, on what basis do they vary? Can Holmes in one world have the nuclear properties that Hamlet has in this world? If not, why not? If so, how?

At one place (p. 187), Parsons gives us a clue as to his doubts over Rigidity. He raises the question of whether Conan Doyle could have written slightly differently about Holmes or if he would thereby have been writing about a different character. Parsons declares himself unable to make up his mind on this question. Now if Conan Doyle could have written differently about Holmes, then, by the Link Hypothesis, Holmes would presumably have had different nuclear properties and so the rigidity assumption would fail.

Such considerations do have some appeal; but it is hard to see how they can be worked up into a viable theory. There is first of all a difficulty in allowing that Conan Doyle might have written differently about Holmes, even if ever so slightly; for a slippery slope argument would seem to yield the counter-intuitive conclusion that Conan Doyle might have set down the text of Hamlet and still have been writing about Holmes. But even if this difficulty is set aside, perhaps as arising from some vagueness as to what object Holmes is, there is the further problem of determining the basis 
upon which variation in nuclear properties is to be allowed. Presumably there are possible worlds in which Holmes is not written about (or otherwise invoked). But then what nuclear properties is Holmes to have in such worlds? It is no use saying "the nuclear properties Conan Doyle actually attributed to him", for that is to exhibit an unjustified bias towards the actual world. Nor can one say "no properties at all", since then distinct nonexistents that were not written about in a given world would turn out to be identical. There are even problems in explaining the variation of nuclear properties in worlds in which the given nonexistents are written about. Following an argument of Dan Hunter's ([3], p. 3), let us suppose that, on twin earth, another author had independently set down the text of the Conan Doyle stories. Then on Parsons' view, Conan Doyle and his twin would have been writing about the same characters, including a detective whom we may call 'Holmes'. Consider now a world in which Conan Doyle sets down a slightly different text, but his twin sets down the same text. Then they have written about distinct characters, say Holmes' and Holmes". But it may plausibly be argued that Holmes' and Holmes" are both identical to Holmes, thereby yielding a contradiction. For what character an author is writing about should not depend upon what other authors independently do. So since each author would have been writing about the same character in either world had the other author been absent, they must each be writing about the same character when the other author is present.

Arguments such as these suggest that the view cannot be worked up into a viable theory. Nor does any other variational view seem plausible; and I therefore propose that the Rigidity Assumption be accepted.

The second question concerns whether objects such as Holmes might have existed. On this question, Parsons again declares neutrality: he is "not at all sure what the right answer should be" (p. 186). But again it seems to me there are compelling reasons for getting off the fence and rejecting the possible existence of such objects as Holmes.

Let us agree to call an object fictitious if it is the kind of object that can be native to a story or other such context. (This is the broad sense of the term. We might reserve the term 'fictional' for the narrow sense of being native to some work of fiction.) It is not necessary that a fictitious object can be native to a story or context, but it must then be of the same kind as other objects that can be so native. So the question is whether fictitious objects can exist. Now in considering such questions, it is often helpful to 
reverse the roles of the actual and the given possible world. So instead of asking whether a fictitious object of this world could be an existent of another world, we ask whether an existent of this world could be a fictitious object of another world. Could you, or I, or the Eiffel Tower, have been the kind of object that is introduced in a work of fiction? When put like this, the answer seems clearly to be no. Surely, even when I do not exist. I am not the kind of object that an author creates in writing a work of fiction. Parsons begins his book with the dedication: To my parents, without whom I might have been one. But this may be a bad beginning; for in the relevant sense of 'non-existent' as fictitious, the dedication would appear to be false.

This intuition is further confirmed by argument. First, the property of being fictitious would appear to be a categorical property of an object. It says what sort of thing the object is and hence applies essentially to it. Now, necessarily, no fictitious object exists. So if a fictitious object could exist, it would still be fictitious and hence not exist. Therefore no fictitious object can exist.

Secondly, consider a fictitious object, such as Holmes, that is introduced in a story or other context. Suppose that Holmes were identical to a possible existent $y$. Now presumably $y$ and the activity by which Holmes is introduced, Conan Doyle's story telling, could both exist in some possible world. It seems reasonable that, in such a world, Holmes should still be introduced in the story, since the relevant activity is the same. But then we have the absurd situation in which an existent is introduced in a story. This absurdity becomes even more apparent if we take the view, later to be defended, that authors literally create the objects introduced in their stories; since then we would have an author creating, through his story telling, an existent character.

But even if one is unmoved by these essentialist considerations, there remains the difficulty of saying, in case of fictitious object can exist, which possible existent it is identical to. Presumably, any such identity must hold in virtue of some sort of agreement between what is true of the fictitious object in its story, should there be one, and what is true of the possible existent in the different possible worlds. But, as several philosophers have pointed out ${ }^{14}$ there is in general no unique possible existent which can be identified with the fictitious object in this way. This difficulty becomes compounded if, as will later be argued for, it is allowed that the same properties may be true of two distinct fictitious objects in their respective stories; for even if we fix on the possible existent, there will still remain an indeter- 
minacy as to which of the fictitious objects it is to be identified with.

To this argument it may be objected that it only shows that a typical fictitious object is not a possible existent, but it leaves open the possibility that every possible existent is a fictitious object. One is inclined to think that if one fictitious object can exist then every fictitious object can exist. But there may be a fundamental difference, though not one of kind, among fictitious objects in this respect.

There have in recent times been two traditions of though that have espoused an ontology of non-existents: the Meinongian; and the modal. The first has found a home for its objects in the intentional - in thoughts, beliefs and dreams; the other has found a home for its objects in the possible - in the unrealized possibilities or possible worlds. For some reason, the modal tradition has been considered more respectable (perhaps because it has a better worked out theory); and so the attempt has usually been to reduce Meinongian objects to possibilia. Against such a reduction, the above argument would appear to succeed. But one might equally well attempt to find a place for the possible within Meinongian ontology; indeed, this appears to be Parsons' strategy. And against such a reduction, the above argument would seem to have little force.

There is, indeed, a specific way in which Parsons might attempt to bring off the reduction of the modal to the Meinongian ontology. He might suppose that each possible existent $y$ is identical to the non-existent $x$ whose nuclear properties are those that $y$ has essentially. This is, in effect to treat existents as if they necessarily had the nuclear properties that they had essentially. There would then be no danger of identifying two possible existents, since each possible existent $y$ would be distinguished by its necessary possession of the nuclear property $\lambda^{N} x(x=y)$ of being identical to $y$.

Although this reduction may be more attractive than the one in the other direction, there are still objections to it and others like it. Suppose that the possible existent $y$ exists in the world $v$, but not in the world $w$. By an innocuous extension of object abstraction, there is an object $x$ that has in $w$ exactly those nuclear properties that $y$ has in $v$. Then $x$ does not exist in $w$; for $y$ will have the property $\lambda^{N} x$ ( $\rho$ is true) in $v$, for any true proposition $\rho$ of $v$, and it is only in $w$ that an existent can have all such properties. It now seems reasonable to conclude that $x$ has in $v$ exactly the nuclear properties that $x$ has in $w$. If $x$ is non-existent in $w$, then this follows by the previous rigidity assumption. On the other hand, if $x$ is existent in $w$, it seems that 
$x$ should at least have in $w$ the nuclear properties that it has in $v$; but then it cannot have more, since $y$ has in $w$ each nuclear property or its nuclear negation, and no existent can have both. Since $x$ has in $w$ exactly the nuclear properties $y$ has in $v$, it follows by Parsons' identity axiom that they are the same. Now the choice of the world $v$ in this argument was arbitrary. As long as $y$ was not a world-bound individual, we could have chosen another world $v^{\prime}$ in which $y$ would have existed and would have had different nuclear properties. But then the same argument would have yielded the identity of $y$ to an object $x^{\prime}$ that had in $w$ exactly the nuclear properties $y$ had in $v^{\prime}$. Since $x$ and $x^{\prime}$ have different nuclear properties, they are distinct. So $y$ is identical to two distinct objects - a contradiction.

The above argument depends upon two rigidity assumptions: the original assumption that a non-existent retains its nuclear properties on staying nonexistent; and the further assumption that a non-existent retains its nuclear properties on becoming existent. Parsons might, of course, reject either assumption. But it would then not be clear what to put in their place. Nor need it be clear how the identification might otherwise proceed. If, for example, the nuclear properties of a non-existent could vary from world to world, there would be no guarantee that a possible existent could be identified with a non-existent on the basis of a fixed class of nuclear properties.

So we see that the possibility of a Meinongian reduction cannot be maintained and that even the possible existence of a special subclass of the fictional objects must be rejected.

If this is done, it will have important consequences both for object theory in general and for Parsons' theory in particular. For one thing, it will now be appropriate to introduce a new predicate, say $\boldsymbol{F}$, for being fictional in addition to the predicate $E$ for existence. Every fictional object will be nonexistent; but not every non existent will be fictional. Thus there will be a tripartite division of the objects, at any one world, into the existent, the fictional, and the possibly existent. We might use the term 'non-existent' for the fictional objects. But then it must be recognised that not every object which does not exist is non existent.

Once the language is expanded in this way, a rigidity assumption for the new predicate should be laid down to the effect that, necessarily, any fictional object is necessarily fictional ( $\square \forall x \square\left(F_{x} \supset \square F_{x}\right)$ ). What this means is that the domain of fictional objects is constant from world to world. Combined with the previous rigidity assumption (with $F$ in place of $-E$ ), 
it has the consequence that, necessarily, any fictional object necessarily has the nuclear properties that it has $(\square \forall x \forall P((F x \wedge P x) \supset \square P x)$. Thus together the two axioms state that each world is the same in regard to the domain of fictional objects and their nuclear properties.

Acceptance of the rigidity assumptions now has consequences for the nonmodal part of the theory. If $y$ is a possible existent that exists in $v$ but not in $w$, there will be an object $x$ that is fictional in $w$ and that has in $w$ exactly the nuclear properties that $y$ has in $v$. By the rigidity assumptions, $x$ will be fictional in $w$ and will have in $w$ the same nuclear properties as $y$.

This will mean, first of all, that the axiom of object identity is too strong: for it will predict that $x$ and $y$ are identical; whereas, since one is fictional and the other not, they are distinct. We can still say that fictional objects with the same nuclear properties are the same; and we can still say that existent objects with the same nuclear properties are the same (which is a consequence, in any case, of the abstraction principles for properties). But what we cannot always do is to distinguish between existent and fictional objects in terms of their nuclear properties. The mere fact that an object has the extra-nuclear property of being existent or being fictional may be required to distinguish it from other subjects.

The example involving $x$ and $y$ also indicates that the abstraction axiom for objects is too weak: for it can only give us some object in $w$ with the nuclear properties possessed by $y$ in $v$; but what we want is a fictional object with those properties. So the abstraction axiom should be stated to read:

For any condition, there is a fictional object whose nuclear properties are those satisfying the condition;

and similarly for the relational forms of the axiom.

It is interesting that similar modifications to the axioms may be prompted by considerations of a different sort altogether. In our discussion of correlates, it was suggested that objects should be capable of a non-circular individuation in terms of their nuclear properties or relations. Now such an extended principle is eminently plausible for fictional objects; but it is highly controversial for existents, involving, as it does, a commitment to a substantial form of Leibniz's principle of the identity of indiscernibles. So we may be lead, through this reason, to restrict the application of the identity axion to fictional objects. Again, in our discussion of the paradoxes, it was suggested that it should only be a set of nuclear properties that defines an object, not an 
arbitrary class. However, it may plausibly be argued that the nuclear properties possessed by an existent should form a proper class. ${ }^{15}$ So the abstraction principle may, for this reason, be strengthened to the claim that each set of properties determines a fictional or, at least, a non existent object.

Finally we may note that, with the modification to the identity and abstraction axioms, Parsons' requirement that the domain of objects be constant from world to world (pp.99-101) is in need of serious qualification. We can take the domain of objects at each world to consist of the existent, the fictional and possible. The domain will then be constant from world to world, but the identity and abstraction principles will not apply in any straightforward way to the possibles. On the other hand, we can take the domain at each world to consist of the existent and the fictional objects. Something like Parsons' principles will then apply to the objects so determined, but the domain will not be constant from world to world.

Parsons falls into the common error of supposing that all non-existents are of the same kind. The attempts to identify fictional with possible objects or possible with fictional objects are, in their own way, also symptoms of this error. It must be recognised, however, that the non-existents do not constitute an amorphous nothingness. Like existents, they come in classes of radically different kinds. They are called upon to play very different roles and, in those roles, are governed by very different principles.

We come now to the radical or external criticisms of Parsons' theory. It will be argued that each of the three main planks of his theory - the abstraction axiom, the identity axiom, and the nuclear/extra-nuclear distinction should go. Fuller versions of the first two criticism can be found in [2], Section $\mathrm{E} 1-2$.

\section{PLATONISM}

Parsons' theory naturally goes with the view that fictitious objects are necessary creatures, that it is not an empirical matter that there is such an object as Holmes. For the only plausible way of maintaining the contrary, given that the quantifiers are to range over what fictitious objects there are, is to have there being a fictitious object dependent upon its being introduced in a story or related context; the object must, as it were, be empirically realized. But the application of object abstraction will generally yield many non-existents that are not in this way realized. 
However, this abstract or platonic conception of fictitious objects runs counter to our ordinary judgement that fictional characters are created by their authors. For to create is to bring into being. So how can an author create a character if the character would have been there regardless of what he did?

Philosophers have usually been uneasy with the ordinary view of the matter; and Parsons is no exception. In talking of an author creating characters, he puts scare quotes around the word (p.51) and refers to a "popular sense" (p. 188). He suggests that what we mean when we ordinarily say that an author creates an object is that he makes it fictional.

But this will not do; to bestow a property on an object that is already there, no matter how honorific the property, is not to create the object. To create is to bring into being. What philosophers such as Parsons must do is to explain how, in making our ordinary judgements about the creation of fictional objects, we are prepared to affirm what is false or prepared to use the word 'create' in a special sense. I myself doubt that any adequate explanation is forthcoming and would prefer to see our ordinary judgements taken as data for our theory to explain.

If we follow this line, then Parsons' abstraction axiom for objects must go. Instead, there being a fictional object must be made to depend upon its being empirically realised. The identity axiom could still be retained. But there would then be an uneasy tension in the theory; for the identity of a fictional object would have one source, its nuclear properties, while the being of the object would have another source, its empirical story or context. It would be preferable to have a unified account of the identity and being of the objects.

The creationist view also makes for difficulties in the modal part of the theory. In our re-styled version of the modal part, we represented the domain of fictitious objects as being constant from world to world. This must now be given up. There will be a fictitious object such as Holmes in the domain of a given world only so long as Conan Doyle (or perhaps another author) does the right things. Thus the objects at any one world will now unfold, according to a fourfold division, into the actually existent, the possibly existent, the actually fictional and the possibly fictional. The category of fictional objects, like the category of existents, will split up into the actual and the possible.

The creationist view, finally, has relevance to an issue that has long bedevilled thinkers in the Meinongian tradition. Do fictional or other non- 
existent objects have any kind of being? Meinong was torn on this question, but tended to the view that they do not (the doctrine of Aussersein). On the other hand, the early Russell thought that non-existents must have some kind of being. In more recent times, Routley [8] has inveighed heavily against the Russellian position, while Parsons declares himself unable to find anything more than a "terminological issue" here (p. 10). On a creationist view, however, there is a clear and compelling reason for ascribing being to fictional objects; for an object could not be created unless it could pass, in some appropriate sense of the term, from non-being to being.

\section{INTERNALISM}

Internalism is the doctrine that fictitious objects can be individuated in terms of their internal content, the properties or relations ascribed to them in the stories or contexts in which they are introduced. Parsons is committed to internalism. For suppose that two distinct objects were indistinguishable in terms of their internal content. By the Link Hypothesis, they could not be distinguished in terms of their nuclear properties; and so by the identity axiom, they would be the same.

There are, however, various objections to the internalist position. One is that two authors could independently write stories with the same content. On Parsons' view, the native characters of either story would be the same; but the intuitive view is that they are not. This intuition is supported by our talk of creation. For each author creates his characters. So assuming one author wrote first, how could the other author create what was already created?

Parsons considers this objection and says, in reply, that the situation is no worse than when two authors independently write (or create) the same story (p. 188). Now there may be a sense of 'story' in which we have the same story here. But what is created, it seems to me, is different for the two authors. Indeed, we can imagine that each story was the start of two quite separate literary traditions. Each tradition would then have its origin in a story created by its own author, but not in the story created by the other author. Thus Parsons' example does not give any support for a sense of 'create' in which we may talk of creating an object that is already created.

Another objection concerns indiscernible objects from within the same story or context. Perhaps the clearest example of this sort is a picture of a symmetric universe. We may suppose that the picture is realised on a surface 
of a sphere and is symmetric about two hemispheres. Each object portrayed on the one hemisphere will be distinct from its counterpart portrayed on the other hemisphere and yet indiscernible within the picture from it.

Parsons considers similar examples in Chapter 7.5 (pp. 190-194), but his ways of getting round them are not really applicable to the present case. We cannot distinguish the objects in terms of the names that they have in the picture (cf. pp. 192-193), since the picture gives no names to the objects. Nor is it part of the picture that this blob portrays this object, that blob the other object. It also cannot be plausibly argued that the picture is really about a pair of objects, but not about its individual members. If there had been a slight discrepancy in the second hemisphere, we would have been under no temptation to say this. But a small variation on the one hemisphere can hardly make a difference as to whether a specific object is portrayed on the other hemisphere.

Another response, not considered by Parsons, is that the one object $x$ has the property of being identical to $x$ in the picture, while the other object $y$ does not. (A similar response might be given in the two authors case.) But against this, it may first be protested that the theory is most reasonably taken to provide a non-circular means of individuating non-existents. Moreover, if the objects $x$ and $y$ could only be distinguished in terms of their identity properties, it would be a complete mystery, as I have already pointed out, why a given blob on the sphere should portray the one object $x$ rather than the other object $y$.

But even without the requirement that individuation be non-circular, there will still be counter-examples against the original form of the identity axiom. Imagine a story in which two characters $x$ and $y$ are symmetrically placed. Although the characters are distinct, it may be left open in the story that they are distinct. It might further be supposed that the story is a logical fantasy in which objects are not self-identical. There would then appear to be no properties at all, circular or not, by which the two objects might be distinguished in the story.

If these examples are accepted, then either the Link Hypothesis or the identity axiom (in its circular or non-circular form) must go. But if the Link Hypothesis goes, we are left with no basis for ascertaining the nuclear properties of fictional objects. Therefore the only plausible alternative is the rejection of the identity axiom. 


\section{LITERALISM}

In most ordinary contexts of discourse, it is acceptable to assert that Sherlock Holmes is a detective, that he lived in Baker Street, and that he smoked opium. According to what I call the literalist view, what makes these assertions acceptable is that they are true. Moreover, these truths are of a straightforward subject-predicate kind. The relevant predicates are simply true of the object named, without any qualification as to the story being implicitly understood and without any special sense being attached to the predicates. ${ }^{16}$

Parsons seems to presuppose the truth of literalism throughout most of the book. But the relationship between his theory and literalism is hard to ascertain, since it is no part of the formal development of the theory that literalism should be true. It may therefore be worthwhile to work out more exactly what the relationship is.

One connection is that the theory may be used to explain the truth of the literalist judgements. Why is it true that Sherlock Holmes is a detective or that he lived in Baker Street? Parsons may supply the answer: because Holmes is the object that it is. The rule for identifying a story object, the Link Hypothesis, takes it to be the object whose nuclear properties are those attributed to it in the story. Since Holmes is said to be a detective in the Conan Doyle story, it will be part of his identity that he is a detective.

However, it seems to me that a much tighter connection can be made out; for something like Parsons' theory seems to be needed to sustain the literalist doctrines. We require, first of all, a general formulation of which literalist judgements are true. Now the immediate basis for the truth of a literalist proposition is the truth of that same proposition (or a related one) in a story or other context. But not all propositions true in a story or context can be taken to be true in reality. For one thing, this goes against the facts: we do not take Sherlock Holmes to exist because he exists in the Conan Doyle stories. But also, it is theore tically untenable: the propositions true in different stories, or even in the same story, may be inconsistent with one another and with what else we take to be true.

So which truths of a story do we take to be exportable into the real world? The only natural answer, at least in the case of propositions of subjectpredicate form, is that it depends upon the object and property referred to. As long as the object is native to the story and the property is of the right 
sort, then the proposition will be exportable; otherwise it will not be (though it may, for other reasons, turn out to be true).

Thus the need to find some barrier to exportation leads naturally to the nuclear/extra nuclear distinction. Indeed, the existence of a barrier can be used as a basis for defining the distinction. For let us say that a property is exportable if any object native to a story or other context will have that property, and will only have that property, in virtue of having it in the story or context. Then the nuclear properties may simply be defined as the importable ones.

It may be a defect of this definition that it uses the phrase 'in virtue of. But in particular applications, the phrase will have a clear meaning; and in most of them, it will be dispensable. For we may take an exportable property to be one that any object native to a story or context has just in case the object has it in the story or context. It is then only in the most freakish or abnormal cases that an object exportable in the one sense will not be importable in the other.

The possibility of defining nuclearity in this way is no small thing. Parsons devotes some effort to explaining the distinction between nuclear and extranuclear properties (pp. 22-26, 72-78, 166-174). But his explanations leave room for considerable doubt over the coherence of the distinction. What we can show is that, once the truth of literalism is granted, a clear and coherent distinction can be drawn along the lines that Parsons intends.

This is not necessarily to say that the distinction is thereby explained in the most fundamental terms. Taking the nuclear properties to be the exportable ones means that the Link Hypothesis becomes a triviality. But presumably we want the nuclear/extra-nuclear distinction to pertain most directly to the intrinsic nature of the properties; it is because of this intrinsic nature that some properties are exportable and others are not. It was perhaps in order to maintain the non-triviality of the Link Hypothesis that Parsons did not avail himself of the above characterization of the distinction (though it may also have been to maintain a neutral position on its truth).

The existence of an exportation barrier gives rise to, and serves to characterize, the nuclear/extra-nuclear distinction. But it does not, in itself, give rise to anything like Parsons' characteristic axioms for the identity and abstraction of objects. These may be seen to arise, not from the demand for a general formulation of which literalist judgements are true, but from the demand 
for an explanation of why they should be true. A native object will have those nuclear properties that it has in its story. But why should it have those properties, and not others?

There are various answers that might be given to this question. But one of the most plausible makes the truth of literalist judgements turn upon the identity of the object in question. Nuclear properties are taken to define nonexistent objects. The rule for determining the identity of an object of a story, in case it is not immigrant, is that it be the object defined by the appropriate cluster of nuclear properties. But this seems to presuppose both that the object is already there (the abstraction axiom) and that it thereby be uniquely determined (the identity axiom). So literalism, while not actually implying a Parsonian theory, gives it a great deal of plausibility.

But what if literalism is taken to be false? How then does the theory fare?

Certainly, it would lose part of its point. Since there would be no literalist truths, there would be no such truths to explain. But still the theory would have some point. The problems of ontology and identity would remain; it would need to be explained which objects there were and what they were.

How the theory would do in these remaining respects would depend critically upon the interpretation of the nuclear/extra nuclear distinction. Suppose the distinction is taken with its intended application to ordinary language, so that predicates like 'is a detective', 'is blue', 'is tall', are taken to be nuclear, while the others, such as 'exists' or 'is mythical', are not. It then seems that the theory must be rejected. For ordinary fictional objects, such as Watson or Holmes, must be distinguished in terms of their nuclear properties. But if literalism were false, it would appear that no nuclear properties could be true of either Watson or Holmes and so there would be no basis for distinguishing them.

On the other hand, the nuclear/extra-nuclear distinction could be regarded in a purely formal light, interpretable in any way consistent with the axioms of a theory. There would then seem to exist two main ways of re-interpreting the distinction - the contextual and the abstract.

On the first, the nuclear weakening $\boldsymbol{P}^{N}$ of $\boldsymbol{P}$ is taken to be the property of an object's either existing and having $\boldsymbol{P}$ or not existing and having $\boldsymbol{P}$ in the context in which it is introduced (cf. pp. 202-203). We may now make some reasonable assumptions about objects and contexts, as in [2]. The main ones, crudely put, are that: 
- For any class of properties, there is an object and a context such that the object is native to that context and has in that context exactly the properties of the class;

- Objects with the same properties in the contexts to which they are native are the same.

Under the proposed interpretation, the basic axioms of the Parsonian theory may then be derived as theorems in the contextualist theory.

But despite the possibility of interpreting the contextualist within the Parsonian theory, there are some basic differences between the two theories under their intended interpretations. First, the two theories will be extended differently. In particular, on the contextual theory, every 'nuclear' property $\boldsymbol{p}^{N}$ should be extra-nuclear, and hence the operation of nuclear weakening is indefinitely iterable; but this is not so on the Parsonian theory. Second, the ultimate basis for the axioms will be different on the two theories. On the Parsonian theory, the principle that an extra-nuclear property agrees with its nuclear weakening on existents represents a fundamental fact either about the operation of nuclear weakening or about the operation of nuclear abstraction, should nuclear weakening be explained in terms of it. But on the contextualist theory, it is triviality under the proposed re-interpretation of nuclear weakening. Finally, and most importantly, the two theories offer very different conceptions of objects and of how the basic problems facing a theory of objects are to be solved. The Parsonian theory conceives of objects as independent of the contexts in which they occur; the basic principles of abstraction and of identity are stated without reference to such contexts. On the other hand, the contextualist theory conceives of objects as locked into the contexts in which they occur; the abstraction and identity principles are stated with essential reference to those contexts.

The other, "abstract", interpretation of the Parsonian theory consists in supposing that all of the properties expressed in ordinary language are extranuclear but that, nevertheless, the nuclear weakenings of those properties exist at an abstract or theoretical level. To understand this interpretation better, suppose that the Parsonian theory correctly applied to ordinary language. Now imagine that all devices for expressing nuclear abstractions or nuclear weakenings were excised from the language and that syntactically simple nuclear predicates were taken to be automatically false of non-existents. In such a language, all nuclear properties would go unexpressed. But from the standpoint of our language, it would be reasonable to suppose that the nuclear 
weakenings of the properties expressed in that language still existed. Transfer now this hypothesis to our actual language, and you have the present interpretation.

Such an interpretation does, indeed, make the Parsonian theory compatible with an anti-literalist stand, at least in regard to the predicates of ordinary language. But it makes the nuclear properties unnecessarily metaphysical. Our only access to those properties is through the role they play in the individuation and determination of objects. But that role is equally well served by the contextualist theory; and so the whole apparatus of nuclear properties can drop out as so much idle machinery. What gives the Parsonian theory the edge over the contextualist theory is its capacity to explain the literalist truths. Without those truths, that explanatory edge disappears.

So we see that there is an intimate connection between Parsons' theory and literalism. If literalism is true, it can be used to sustain the nuclear/extranuclear distinction and provides some evidence for the rest of Parsons' theory. If literalism is false, then the nuclear/extra-nuclear distinction, as it is ordinarily understood, must go. The only way to save the Parsonian theory is to subject the distinction to radical re-interpretation, either by postulating nuclear properties at the purely metaphysical level or by conceiving them in contextualist terms.

In the light of its importance, both for Parsons' theory and object theory in general, it is surprising that the doctrine of literalism has not been discussed further. Parsons says little (pp. 53-54), and others not much more. This is hardly the place to debate its merits, but I can briefly hint at the reasons I think the doctrine false. The central question is whether ordinary predicates such as 'detective' or 'dagger' can sometimes be true of non-existents. A great deal of the linguistice evidence favours the literalist view; but there is some with which it is hard to reconcile. Macbeth asked: "Is this a dagger that I see before me?" Now, assuming this is a situation from real life, it seems reasonable to suppose that Macbeth could have been wrong in his answer to this question, that if he had in the perceptual situation, taken the object that he saw to be a dagger, then his belief would have been mistaken. But on literalist views, it is hard to see how Macbeth could have made a mistake. If he had taken the object to be a dagger then, by the appropriate form of the Link Hypothesis, applied now to perceptions, the object would have been a dagger and so his belief that it was a dagger would have been correct.

The literalist might say, in response to this example, that the word 'dagger' 
is being used in a special sense, to mean what we would ordinarily mean by 'real dagger'. Now there is, indeed, a use of the term 'dagger' that seems to favour the literalist's case. Even if Macbeth is apprised of the true state of affairs, he might say, on a recurrence of the incident: "I saw that dagger again." But it seems to me that it is this use of the word that should be regarded as special, and not the other; it is this use that would be guarded by scare-quotes, if either were.

If Parsons' theory fails on these three counts of platonism, internalism and literalism, what is to be put in its place? My own view might be summed up in the words: fictitious objects are essentially objects of reference. This means, first of all, that there can be no fictional object without a mark or symbol by which it is introduced. It means, secondly, that fictional objects are essentially tied to those marks - no fictional object without the particular mark by which it is introduced. Such a view avoids the pitfalls of Parsons' position. It fits in well with creationist and externalist doctrines: for we may say that a fictitious object is created through its mark being created; and we may allow that fictitious objects with the same internal content should be introduced by different marks. The view, though neutral on literalism, is not required to give it a central place in the determination and individuation of fictitious objects; literalism, if true, may be regarded as a quirk of language without any essential bearing on the identity of objects. Finally, the view is suggestive of an anti-realist position, one according to which features of fictitious objects are ultimately to be explained in terms of features of their marks.

\section{NOTES}

* I should like to thank Terence Parsons for his helpful comments on an earlier draft of this review.

1 Others include Castañeda [1], Rappaport [7], Routley [8] and Zalta [9].

2 These two approaches are also described in Chapter 1.2 of Zalta's [9].

3 The reader should note that I have departed from Parsons' notation in two respects. I have used capitals, rather than lower-case letters, for nuclear relations; and I have used $\boldsymbol{R}^{N}$ or $\boldsymbol{R}$, instead of $w(R)$, for the nuclear weakening. I find my own notation easier on the eye.

${ }_{4}$ I here must assume that $\lambda^{N_{x}(x)}=\lambda^{N_{x \psi}(x)}$ if $\lambda x \varphi(x)=\lambda x \psi(x)$. If the definition is to be applicable to all properties, I must also assume that each property is of the form $\lambda x \varphi(x)$.

In the sequel, I shall tacitly make these and related assumptions. However, the whole topic of the identity of properties is a vexed one and cannot properly be dealt with here. 
3 This, in its turn, follows from the assumption that each nuclear property $P$ is of the form $\lambda N_{x \varphi}(x)$; for then $P$ is the nuclear weakening of $\lambda x \varphi(x)$.

6 Again, the correctness of this definition depends upon certain assumptions. Its universal applicability also depends upon the assumption that all properties are of the form $\lambda x \varphi(x)$. In making this intuitive distinction, I appear to be disputing the theory of property identity briefly proposed on pp. 109-110.

7 The symbolic formulation of the statement is $\exists x \exists y \exists R \forall P[(P x \equiv P=[R y]) \wedge(P y \equiv$ $P=[x R])]$. I omit the proof of its underivability. Parsons (p. 194) claims that the related formula $\exists x \exists y \exists R(x \neq y \wedge \sim E ! x \wedge \sim E ! y \wedge x[R y] \wedge[x R] y)$ is underivable. This is true, even when the inner conjuncts $\sim E ! x$ and $\sim E ! y$ are dropped. It is, however, a rather trivial defect in the system and arises merely from the fact that the system cannot prove the existence of two nuclear properties. If there were only one nuclear property, $x[R y] \wedge[x R] y$ would imply $x=y$, since $x$ and $y$ would then both possess all nuclear properties. On the other hand, under the assumption that there are two nuclear properties (which follows from there being at least one existent), Parsons' formula can be derived. (Let ' $x$ ' have all nuclear properties and let ' $y$ ' have just $[x R]$ as a nuclear property.) Our own underivability result points to a more fundamental defect in the system and cannot be mended by means of such a simple extension to its axioms.

${ }^{8}$ A general case might be made out for adding such a primitive to theories of properties and the like. One could then define interesting notions, such as purity, and formulate interesting principles concerning objectual content, without going to the length of giving a full account of the identity of the intensional entities in question.

9 Let $x S \operatorname{SOr}_{R} y$ abbreviate $\forall P[(P x \equiv P=[R y]) \wedge(P y \equiv P=[x R])]$. Then the formula $\forall R \forall x, y, u, v\left[\left(x \neq y \wedge u \neq v \wedge x \operatorname{SCor}_{R} y \wedge u \operatorname{SCor}_{R} y\right) \supset(x=u \wedge y=v)\right]$ cannot be proved within Parsons' system.

10 I do not have an answer to Parsons' 'exercise' on p. 238. But if there were an interpretation of the stipulated sort, it could not contain a predicate constant satisfying the conditions required of conceivability; for in view of our revised formulation of the paradox, there can be no predicate constant $C$ for which $\forall x \forall y(C x \wedge C y \wedge x \neq y \supset$ $[R x] \neq[R y])$ is true in the interpretation for any given predicate constant $R$. For this reason, the existence of the given interpretation is hardly sufficient to establish the consistency of Parsons' proposal.

11 A similar criticism applies to the system constructed in [9]. Even if the system should turn out to be consistent, it is still not clear what the underlying conception of objects is.

12 Indeed, at one point (p. 234), he says that one must "either maintain that $g$ [the function from $x$ to $[R x]]$ is not, after all, a one-one function, or give up the correlation of objects with sets of nuclear properties". But he means, of course, not sets, but classes or conditions.

13 I am actually interested in the stronger claim that $\square \forall x \square \forall P \square((\sim E x \wedge P x) \supset$ $\square(\sim E x \supset P x))$. But as is usual in the discussion of modal matters, such subtleties will be ignored.

14 See Kripke [5], pp. 763-765, Kaplan [4], pp. 505-506, and Plantinga [6], pp. 154-155. Strictly speaking, Kaplan and Plantinga only argue against the linguistic thesis that fictional names refer to possible existents, but their arguments may be modified so as to apply to the ontological thesis that fictional objects are not identical to possible existents.

is The argument goes as follows: the nuclear properties must form a proper class, or else the paradoxes could be derived. Now each existent will have a nuclear property ${ }_{\lambda} N_{X \varphi}(x)$ or its internal negation $\lambda_{x} \sim \varphi(x)$. Granted that the negations of distinct nuclear properties are distinct, there will then be a one-one correspondence between the nuclear properties possessed by the existent and all nuclear properties. Therefore the nuclear properties possessed by the existent will also form a proper class. 
${ }_{16}$ Crispin Wright has pointed out to me that this formulation of the issue presupposes that the anti-literalist make use of an external concept of truth. For even he would admit that when it is acceptable to say that Holmes is a detective, there is a sense of 'true' in which it is also acceptable to say that it is true that Holmes is a detective. However, I shall not consider to what extent the issue can be formulated without using the external concept of truth.

\section{BIBLIOGRAPHY}

[1] Castañeda, H. N.: 1974, 'Thinking and the structure of the world', Philosophia 4, pp. 3-40.

[2] Fine, K.: 1982, 'The problem of non-existents, I - internalism', Topoi 1, pp. 97 140 .

[3] Hunter, D.: 1981, Reference and Meinongian Objects, Grazer Philosophische Studien, Vol. 14, pp. 23-36.

[4] Kaplan, D.: 1973, 'Bob and Carol and Ted and Alice', in Approaches to Natural Language, ed. by J. Hintikka, J. Moravcsik, P. Suppes (D. Reidel, Dordrecht).

[5] Kripke, S.: 1972, 'Naming and necessity', in Semantics of Natural Language, ed. by D. Davidson and G. Harman (D. Reidel, Dordrecht), pp. 178-214.

[6] Plantinga, A.: 1974, The Nature of Necessity (Oxford University Press, London).

[7] Rappaport, W.: 1978, 'Meinongian theories and a Russellian paradox', Noûs 12, pp. $153-180$.

[8] Routley, R.: 1980, Exploring Meinong's Jungle and Beyond (Australian National University, Canberra).

[9] Zalta, E. N.: 1980, 'An introduction to a theory of abstract objects', Ph.D. Thesis (University of Massachusetts, Amherst).

Department of Philosophy,

University of Michigan,

Ann Arbor, MI 48109.

U.S.A. 